\title{
Exploring Low-Energy Pathways that Interconvert the Apo and Bound States of a Metabolite-Sensing Gene-Regulatory RNA Switch
}

\author{
Joseph E. Wedekind*, Debapratim Dutta, Chapin E. Cavender, Andrew M. DiCola, Griffin M. \\ Schroeder, David H. Mathews and Jermaine L. Jenkins
}

Department of Biochemistry \& Biophysics, and Center for RNA Biology, University of Rochester School of Medicine and Dentistry, Rochester NY 14642, USA.

${ }^{*}$ Corresponding author: e-mail: joseph.wedekind@rochester.edu

Non-protein coding RNA is a central player in biology that has upended old rules naming proteins as the essential factors of gene regulation. Riboswitches are prime examples of RNA sensors that directly bind metabolites to control downstream genes. Although numerous riboswitcheffector complexes have been determined, a central challenge is to understand how such molecular recognition confers conformations that govern transcription or translation. To address this question, we determined new structures of a bacterial riboswitch in the presence and absence of its cognate metabolite, $\operatorname{pre}_{1}$ (Panel 1). A structural comparison reveals major differences in the effector-binding pocket, where $\mathrm{Mn}^{2+}$ ions stabilize different conformations of the $\mathrm{L} 2$ ligandrecognition-loop (Panel 2). The observation that L2 is now well-defined in both structures allowed us to analyze low-energy pathways describing the interconversion of apo and bound states using nudged elastic band calculations. These transitions reveal how Ade14 moves out of the apo-state binding pocket as Cyt 15 enters to recognize pre $_{1}$. Such pathways are supported experimentally by preQ $_{1}$-dependent changes in: (i) riboswitch methylation at adenine and cytosine, and (ii) fluorescence quenching of 2-aminopurine substituted at position 14. A key finding is that the distant and solvent exposed Shine-Dalgarno sequence becomes sequestered and stabilized during the apo-to-bound state transition by packing against reorganized L2 bases Ade13, Ade 14 and Cyt15, which form a three-tiered base stack. The results provide unprecedented insight into how effector sensing induces long-range structural changes leading to gene regulation. Details of the conformational pathways will be discussed in light of supporting experimental data.

Panel 1 | PreQ $_{1}$ is an intermediate in $Q$ biosynthesis

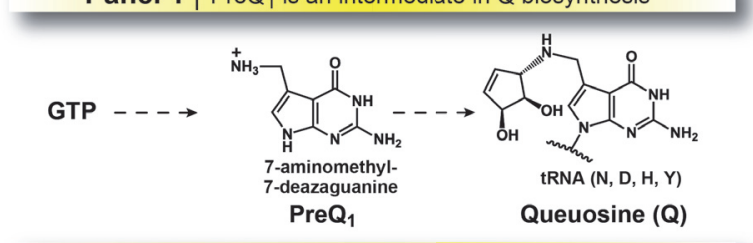

Panel 2 | L2 loops of apo- and bound-state riboswitch structures

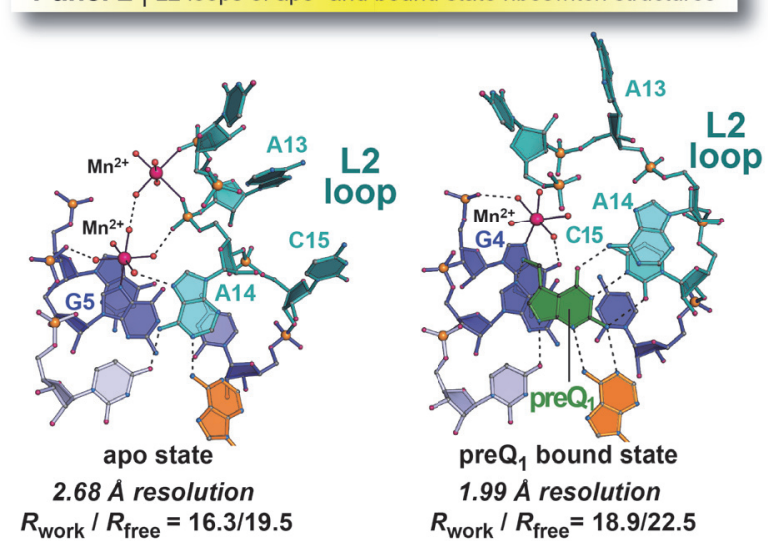

